\title{
Development of a disposable paper-based potentiometric immunosensor for real-time detection of a foodborne pathogen
}

\author{
Nádia F.D. Silva ${ }^{\mathrm{a}, \mathrm{c}}$, Cláudio M.R. Almeida ${ }^{\mathrm{b}, 1}$, Júlia M.C.S. Magalhães ${ }^{\mathrm{b}, *}$, Maria P. Gonçalves ${ }^{\mathrm{b}}$, \\ Cristina Freire ${ }^{c}$, Cristina Delerue-Matos ${ }^{a}$ \\ ${ }^{a}$ REQUIMTE/LAQV, Instituto Superior de Engenharia Do Porto, Politécnico Do Porto, Rua Dr. António Bernardino de Almeida 431, 4200-072, Porto, Portugal \\ ${ }^{\mathrm{b}}$ REQUIMTE/LAQV, Departamento de Engenharia Química, Faculdade de Engenharia, Universidade Do Porto, Rua Dr. Roberto Frias, 4200-465, Porto, Portugal \\ ${ }^{\mathrm{c}}$ REQUIMTE/LAQV, Departamento de Química e Bioquímica, Faculdade de Ciências, Universidade Do Porto (FCUP), Rua Do Campo Alegre, S/n, 4169-007, Porto, \\ Portugal
}

\begin{abstract}
A b s t r a c t
This work reports a new paper-based sensing platform and its application in a label-free potentiometric immunosensor for Salmonella typhimurium detection based on the blocking surface principle. A paper-based strip electrode was integrated with a filter paper pad which acted as a reservoir of the internal solution. The design offers a convenient platform for antibody immobilization and sampling, proving also that is a simple and affordable methodology to control an ionic flux through a polymer membrane.

Two different immunosensing interfaces were assembled on the developed paper-strip electrode. The simplest interface relied on direct conjugation of the antibody to the polymer membrane and the second one resorted to an intermediate layer of a polyamidoamine dendrimer, with an ethylenediamine core from the fourth generation. Electrochemical impedance spectroscopy was used to assess the successive interface modification steps and the resulting analytical performance of both immunosensors was compared. For such, the potential shift derived from the blocking effect of the ionic flux caused by antigen-antibody conjugation was correlated with the logarithm of the Salmonella typhimurium concentration in the sample. In optimized conditions, a limit of detection of 5 cells $\mathrm{mL}^{-1}$ was achieved. As a proof-of-concept, the proposed method was applied to apple juice samples, demonstrating to be a suitable prototype to be used in real scenarios in useful time ( $<1 \mathrm{~h}$ assay).
\end{abstract}

Keywords:

Paper-based

Immunosensor

Potentiometry

Label-free

Foodborne pathogens

\section{Introduction}

The detection and effective prevention of foodborne illnesses caused by bacteria still stand today a worldwide public health issue. Despite this topic being of great interest to public health and the constant demanding to create analytical tools that achieve the analytical performance of conventional plate count techniques, those remain along the years as the gold standard methods since they fulfil the limits of detection imposed by the European Union (EU) regulation 2073/2005 (European Commission , 2005) and the standard ISO 6579-1 (Standardization 2017) to food quality assessment. Among other bacterial pathogens, Salmonella spp. was one of the most common causes of foodborne outbreaks, originating many hospitalizations and deaths every year (Silva et al., 2018; Srisa-Art et al., 2018). According to the summary of foodborne outbreaks in EU occurred in 2016 reported by the European Food Safety Agency (EFSA) and the European Centre for Disease Prevention (ECDP) an unexpected increase of $11.5 \%$ compared to 2015 data (EFSA and ECDP 2017) was observed and the last summary steps up the increasing tendency of the last years (EFSA and ECDP 2018). By the dangerousness of salmonellosis side-effects (especially in typhoid salmonellosis), a tight limit of a single bacterial cell in a $10-25 \mathrm{~g}$ of the sample was imposed, meaning that the presence of a single cell will determine the rejection of the product (EC No, 20173/ 2005, 2005).

Although, besides the need to attain to a sensitive method, other features like the speed of analysis, cost, portability, the complexity of the procedures and equipment accessibility must be considered given that most of the infection outbreaks occur in developing countries and the cost-effectiveness of the methods is the first barrier to industrial acceptance. Accordingly, in last years a great demand for lab-on-chip infield testing devices for real-time detection of foodborne pathogens has been observed, in which electrochemical biosensors reached a 
prominent place by rapidness, sensitivity, portability, low-cost and user-friendly interface which have shown. Despite this, a perfect method that can be used onsite in different matrices is still needed (Silva et al., 2018).

Important goals have been achieved with the developed electrochemical biosensors. Recently, some potentiometric (Hernández et al., 2014; Zelada-Guillén et al. 2009, 2013) and impedimetric (Ranjbar et al., 2018) label-free aptasensors such as a amperometric genosensor (Liébana et al., 2009) presented capabilities to detect a single cell in a small buffered sample volume in a few minutes. Despite this important outcoming in rapid zero tolerance electrochemical biosensing systems, the final product is still expensive due to the high cost of the electrodes and bioreceptors. So, in our view, the upcoming developments must be directed to using low-cost materials for electrode construction and sensors miniaturization for a low-scale use of chemical and biological reagents.

Filter paper has been extensively used as a substrate or mechanical support of analytical platforms due to its flexibility, biodegradability, biocompatibility, portability, low-cost and decentralized accessibility (Ruecha et al., 2017). Recent paper-based biosensors have been used mainly together to colorimetric or electrochemical detection in test strips format (Hossain et al., 2012), microchips (Khan et al., 2018) or microfluidics devices (Altintas et al., 2018), with obvious focus on point-of-care disposable sensors (Narang et al., 2017; Pavithra et al., 2018; Teengam et al., 2017; Tian et al., 2017; Wang et al., 2016) and environmental monitoring (Apilux et al., 2010). Although, this type of devices for foodborne pathogens detection are scarce in the literature (Bhardwaj et al., 2017; Srisa-Art et al., 2018). Despite the appealing output of the existing colorimetric methods, the paper-based electrochemical biosensors for pathogen detection also offer the possibility to quantify the target analyte in useful time, with results similar to the conventional electrochemical schemes (Bhardwaj et al., 2017; Liu et al., 2014). Additionally, potentiometric techniques demonstrated an unparalleled simplicity, portability and low-cost instrumentation (Novell et al., 2012), but paper-based platforms for foodborne pathogens detection with potentiometric detection is still to be explored. Hereupon, the possible interferences from matrix components and the need for a low ionic-strength media is still a big challenge of the electrochemical detection techniques applied to foodstuffs.

In this work, a simple and cost-effective paper-based potentiometric immunosensing platform was developed and a label-free potentiometric immunosensor for Salmonella thyphimurium (ST) detection, based on surface blocking principle (Gyurcsanyi et al., 2003; Pawlak et al., 2015; Silva et al., 2019; Xu et al., 2005) is presented as a proof-of-concept. This detection principle comprises the regulation of a marker ion flux from or into an ion selective membrane mediated by a specific biorecognition event that takes place near or on its surface, which is able to induce a potentiometric response, which can be used as analytical signal. In the present case, the disruption of internal solution flux to through the sensing membrane towards the bulk solution (sample side) is disrupted by the binding of ST cells to the immunosensing interface (Ozdemir et al., 2013; Pawlak et al., 2015). Thereby, inspired in the newest paper-based ion-selective potentiometric sensors (Armas et al., 2018; Hu et al., 2016; Rius-Ruiz et al., 2011; Ruecha et al., 2017; Yoon et al., 2017), a common filter paper strip soaked with a poly(3, 4ethylenedioxythiophene)polystyrene sulfonate (PEDOT:PSS) conducting polymer was used as ion to electron transducer in the indicator electrode, on which it was dropped a thin flexible polymeric film which serves both as ion selective membrane and as platform for bio-recognition element loading.

The detection of biomolecules or larger analytes by paper-based analytical devices frequently involves elaborated construction schemes, resorting to self-assembled monolayers (SAMs) or nanomaterials for bioreceptor anchoring, concerted with enzyme or nanoparticle labeling to reach to desired low detection limits, increasing the cost and complexity of the methods (Canovas et al., 2017; Parrilla et al., 2017; Srisa-
Art et al., 2018). Here, the polymer membrane casting step allowed the introduction of a cation selective membrane with available carboxylic acid groups allowing the covalent attachment of the antibodies without the need of extra elaborated chemical steps for cellulose paper modification (Cao et al., 2017). In turn, the materialization of surface blocking concept as a detection method of the proposed paper-based immunosensor was made resorting to a passive transmembrane flow of a reporter ion from the back of the polymer membrane to the place where the desired confined surface immune reaction happens (Pawlak et al., 2015). For that, a small filter-paper pad which serves as a reservoir of reporter ion solution was placed on the back of the electrode, mimetizing and transforming it in a "vertical ion flow" device.

Considering the effect on the biosensor performance of a stable, strong and oriented immobilization of antibodies (Fu et al., 2005; Yang et al., 2018) two immunosensing interfaces (IMS) were studied. The simplest IMS relies on direct conjugation of the antibody to the carboxyl groups present on polymer membrane backbone by an EDC/NHS covalent coupling chemistry. The second one resorts to a polyamidoamine dendrimer intermediate layer, with an ethylenediamine core from fourth generation (PAMAM $\left(\mathrm{NH}_{2}\right)$ 64dendrimer) in an attempt to increase the available area to antibody immobilization.

The proposed immunosensor was successfully applied, and the results show that it can accurately predict low levels of ST with high specificity. Some limitations and prospects for use of this electrode prototype in mass-scale and real scenarios are discussed.

\section{Experimental}

\subsection{Chemicals and solutions}

ST positive control $\left(5 \times 10^{9}\right.$ cell $\left.\mathrm{mL}^{-1}\right)$ was obtained from Kirkegaard \& Perry Laboratories. The Salmonella monoclonal antibody $\left(100 \mu \mathrm{gL} \mathrm{m}^{-1}\right)$, highly specific for surface liposaccharides of the typhimurium serotype (Freitas et al., 2014; Viswanathan et al., 2012), were purchased from Santa Cruz Biotechnology.

Bovine Serum Albumin (BSA), sodium dihydrogen phosphate dihydrate $\left(\mathrm{NaH}_{2} \mathrm{PO}_{4} \cdot 2 \mathrm{H}_{2} \mathrm{O}\right)$, potassium hydrogen phosphate $\left(\mathrm{KH}_{2} \mathrm{PO}_{4} \mathrm{xH}_{2} \mathrm{O}\right)$, 4-morpholineethanesulfonic acid hydrate (MES), hydrochloric acid $(\mathrm{HCl}), \mathrm{N}$-(3-Dimethylaminopropyl)-N'-ethylcarbodiimide hydrochloride (EDC), N-hydroxysuccinimide (NHS) and PAMAM $\left(\mathrm{NH}_{2}\right)_{64}$ dendrimer (10 $\mathrm{wt} \%$ in methanol) were from Sigma Aldrich. Poly(vinylchloride) carboxylated (PVC-COOH, 1.8\%) and 2-nitrophenyl octyl ether (2-NPOE) were Selectophore ${ }^{\mathrm{TM}}$ grade from Sigma Aldrich. All other reagents used throughout this work were pro-analysis quality (pa) or equivalent and were used as received.

The polymer membrane sensor cocktail was obtained simply by mixing the plasticizer 2-NPOE $(\approx 66.6 \mathrm{wt} \%)$ and PVC-COOH $(\approx 33.4 \mathrm{wt} \%)$ dissolved in tetrahydrofuran (THF).

Phosphate buffer saline (PBS) $0.01 \mathrm{~mol} \mathrm{~L}^{-1} \mathrm{pH} 7.4$, prepared with $0.1 \mathrm{~mol} \mathrm{~L}^{-1} \mathrm{Na}_{2} \mathrm{HPO}_{4}$ and $0.1 \mathrm{~mol} \mathrm{~L}^{-1} \mathrm{KH}_{2} \mathrm{PO}_{4}$, was used for ST positive control dilutions. For antibody dilution, PBS $0.1 \mathrm{~mol} \mathrm{~L}^{-1} \mathrm{pH} 7.4$ with a background of $25 \mathrm{mmol} \mathrm{L}^{-1}$ of sodium was used. Phosphate buffer (PB) $0.01 \mathrm{~mol} \mathrm{~L}^{-1} \mathrm{pH} 7$ was prepared with $\mathrm{NaH}_{2} \mathrm{PO}_{4} \cdot 2 \mathrm{H}_{2} \mathrm{O}$ and the $\mathrm{pH}$ was adjusted with $\mathrm{NaOH} 4 \mathrm{~mol} \mathrm{~L}^{-1}$.

\subsection{Fabrication of paper-based immunosensor}

The general procedure for immunosensor fabrication involves the construction of the paper-strip electrodes (PSE) (Fig. 1), followed by the modification of the PSE with the capture antibody (Fig. 2).

\subsection{Construction of paper-strip electrodes}

The filter paper was coated with PEDOT: PSS conductive polymer doped with $10 \%(\mathrm{v} / \mathrm{v})$ of ethylene glycol and was placed in the oven, at $105^{\circ} \mathrm{C}$ for about $7-8 \mathrm{~min}$ to promote solvent evaporation and to 

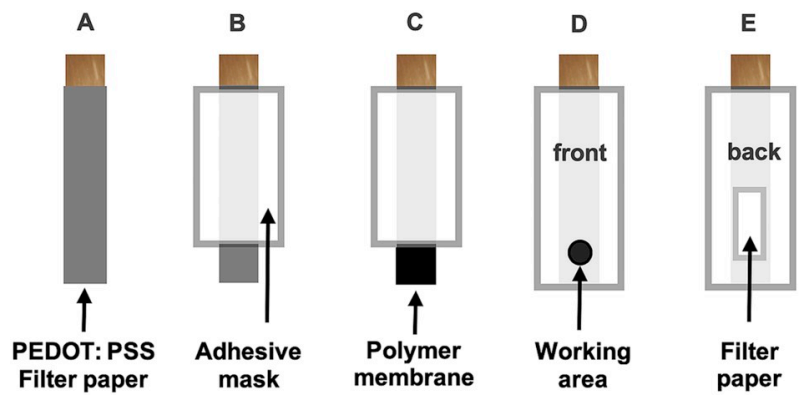

Fig. 1. Schematic representation of the paper-strip micro-electrode construction.

improve the electrical conductivity properties. The amount of conductive ink applied was $35 \mu \mathrm{L} \mathrm{cm}^{-2}$ and the obtained conductive paper showed a resistivity around $6 \mathrm{~kW} \mathrm{~cm}^{-1}$. Then, $5 \mathrm{~mm}$ wide $\times 40 \mathrm{~mm}$ long strips were cut (Fig. 1A) and were sandwiched between two adhesive tape masks, leaving an end of the PEDOT: PSS filter paper $(5 \times 5 \mathrm{~mm})$ and the wire connection zone previously covered with copper tape (Fig. 1B). Then the PVC-COOH based membrane was dropcast of the on the non-covered area (C). For that, $35 \mu \mathrm{L}$ of membrane cocktail were dropped in five successive additions, $7 \mu \mathrm{L}$ at once, three in the front and two in the back of the conductive paper strip. A waterproof material was obtained following this procedure because the conductive paper microfibers were embedded by a PVC-COOH polymer membrane. The last two steps were performed to insulate the electrode body. A circular window with $2 \mathrm{~mm}$ diameter, which was the active surface area of the biosensor, was exposed on the front of the electrode (D). A small strip of filter paper, which served as a reservoir for the internal solution, was placed on the backside of the sensor membrane and the PSE was sealed with insulating tape (E).

\subsection{Biofunctionalization of the paper-strip electrodes}

The functionalization of PSE with the capture antibody was made resorting to two different IMS configurations, represented in Fig. 2. In IMS1, the covalent attachment of ST antibodies was made using the carboxylic groups in the polymeric membrane exposed in the front of the bare PBE. As the $O$-acylisourea formed by the reaction of EDC alone is very unstable, a carbodiimide coupling protocol using EDC along with NHS reagent was used, whereupon the NHS-ester is more stable at physiological pH values. A two-step protocol was used to prevent selfconjugation between the antibodies (Booth et al., 2015). The mixture was prepared in a molar ratio of 1:7 (EDC: NHS) in $0.1 \mathrm{~mol} \mathrm{~L}^{-1} \mathrm{MES}$ buffer at pH 6 and used just after its preparation in a 20 min activation protocol. The excess of EDC was eliminated with two washing steps, with MES and water respectively. Next, the antibody loading was promoted by the incubation of the antibody against ST (Ab) trough dropcast of an Ab solution (diluted 1:100 in PBS pH 7.4, $1 \mu \mathrm{gL}^{-1}, 20 \mu \mathrm{L}$ ) for $2 \mathrm{~h}$, at $4{ }^{\circ} \mathrm{C}$ in a moist atmosphere.
In a second approach (IMS2), a PAMAM(NH$\left.{ }_{2}\right)_{64}$ dendrimer was also used in the immunosensing interface. Herein, the PAMAM $\left(\mathrm{NH}_{2}\right)_{64}$ dendrimer ( $0.5 \mathrm{wt} \%$ in MES buffer) was coupled with carboxylic groups in the polymer membrane through EDC/NHS in a two steps reaction, in similar conditions previously used for antibodies load. The second modification step was performed resorting to glutaraldehyde, which is a bidirectional crosslinker, can react with the terminal primary amines from a PAMAM $\left(\mathrm{NH}_{2}\right)_{64}$ dendrimer and the amine moieties from antibodies. At optimized conditions, $20 \mu \mathrm{L}$ of glutaraldehyde solution (1 wt $\%$, in $0.01 \mathrm{M}$ PBS pH 7.4) was cast on the sensor membrane and reacted for 10 min Following a washing step with MES and incubation with the

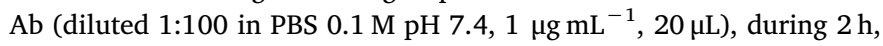
at $4{ }^{\circ} \mathrm{C}$ in a moist atmosphere. Afterwards, to avoid non-specific interactions in both configurations, a BSA solution (1\% in $0.01 \mathrm{M} \mathrm{PBS} \mathrm{pH}$ 7.4, $20 \mu \mathrm{L}$ ) was cast on the sensor surface and allowed to react for $30 \mathrm{~min}$. A washing step with PBS at the end of these two final steps was taken.

Since the modification steps were performed, the electrodes were filled (in the backside) with a tetrabutylammonium chloride (TBACl) solution $(14.4 \mathrm{mM}$ in $\mathrm{PB} \mathrm{pH} 7, \approx 100 \mu \mathrm{L})$ until the paper filter stays bridged by the solution and were kept at $4{ }^{\circ} \mathrm{C}$ in a moist atmosphere until use. These parameters were chosen upon experimental optimization of the sensor response towards TBACl using both conventional and paper-based sampling, in parallel with stir and conditioning effect studies (Supporting Information, S1).

\subsection{Immunosensing interface characterization}

The structural and morphological features of the developed PSE were characterized by Scanning Electron Microscopy (SEM). The SEM studies were carried out at CEMUP (Centro de Materiais da Universidade do Porto), Porto, Portugal. The SEM images were obtained with a scanning electron microscope Quanta 400 FEG scanning electron microscope, (SEM, FEI, Hillsboro, OR), operated in high vacuum/secondary electron imaging mode using an accelerating voltage of $10 \mathrm{kV}$ and working distances of $9.8 \mathrm{~mm}$. Elemental analysis was performed using the same scanning electron microscope coupled with an energy dispersive spectroscopy (EDS) operated at $15 \mathrm{KV}$ with a detector type SUTW SAPHIRE analysis system of resolution 132.19 .

The electrochemical behaviour and step-by-step modification protocol were accessed by faradaic electrochemical impedance spectroscopy (EIS). Experimental details were presented in section S.3 in Supporting Information.

\subsection{Potentiometric measurements}

The electromotive force (EMF) was measured with an EMF 16 Interface (Lawsons Labs Inc., Malvern) and recorded automatically with the provided software. A double junction commercial reference electrode (Orion 90-02-00) from Thermo Scientific Orion with 0.01 M PB $\mathrm{pH} 7$ solution in the outer compartment was used. All measurements

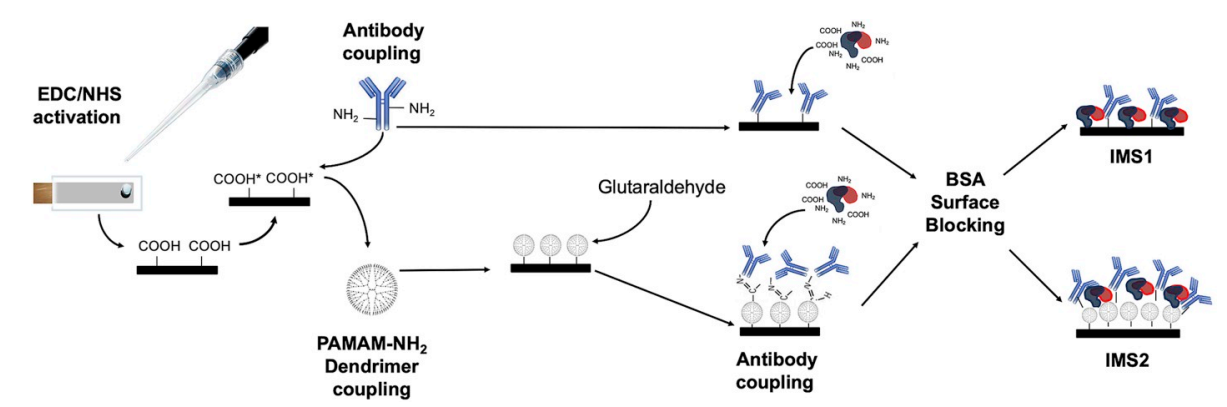

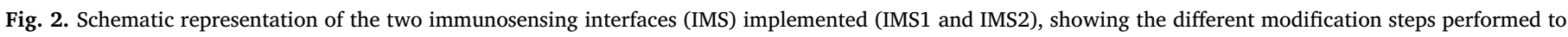
antibody coupling and electrode surface blocking. 
were performed at room temperature $\left(\approx 23^{\circ} \mathrm{C}\right)$ in a faraday cage. A magnetic micro-stirrer from Velp was used (150 rpm).

Potentiometric response of the immunosensor towards IgG antigen is based on the EMF change $(\Delta \mathrm{E})$ induced by the antigen-antibody surface confined reaction. Control and real sample evaluations were performed measuring the difference between the steady-state potential obtained in a blank solution ( $\mathrm{PB}, \mathrm{pH} 7$ ) - named as baseline potential and the potential shift obtained in the presence of a certain amount of ST cells or non-spiked solutions (positive control or negative controls). Calibration curves were obtained by the standard addition method and presented as the average of three different inter-assay replicas with the respective standard deviation. The analytical limit of detection (LOD) was calculated as $3 \sigma$ of the baseline noise of at least three replicates experiments. Additionally, the "smallest amount of analyte in the sample that can be reliably distinguished from zero" was experimentally attained, according to the IUPAC guidelines (Currie, 1995) and ISO 11843 ((ISO) 1997, 2003, 2008).

\subsection{Juice sample analysis}

Apple juice samples purchased in a local supermarket were tenfold diluted with water and artificially contaminated with different amounts of ST cells. Then, a protocol based on filtration through a sterilizing syringe filter $(0.2 \mu \mathrm{m}$ pore size) followed by one washing ( $100 \mathrm{~mL}$ of $\mathrm{PB}$, once) and elution step ( $5 \mathrm{~mL}$ of PB) were taken (Zelada-Guillén et al. 2010, 2013). Finally, the potentiometric immunosensors were used for ST cells detection and quantification in the eluate. Following this procedure, three samples (2 replicas each) with ST cells concentration up to critical infection dose level $\left(50,100\right.$ and 1000 cells $\left.\mathrm{mL}^{-1}\right)$ and control samples (non-spiked) were analyzed.

\section{Results and discussion}

\subsection{Immunosensor development}

The development of this label-free immunosensor involves several challenges related to immunosensor design, the development of a reproducible construction procedure of the PSE base sensor, the biofunctionalization of the sensor membrane with an appropriate immunosensing interface and the general assay conditions. All these parameters must be in tune in order to achieve a sensitive and robust analytical method.

\subsection{Paper-based cation-selective electrode construction}

Most of the common zero-current potentiometric techniques resort to highly specific ion selective electrodes (ISE) able to detect changes in ion activities between their phase boundaries (Xu et al., 2005). In these simplest cases, the response follows the Nernst equation independently of the adsorption phenomena at the membrane surface. Conversely, this work intends to use a label-free sensing mechanism based on the blocking surface principle whereupon a controlled ionic flux is disturbed by a biorecognition event at an ISE polymer membrane surface, triggering an EMF change (Gyurcsanyi et al., 2003; Pawlak et al., 2015). Hence, the PSE development faces an extra challenge of maintaining the practical and functional design of all solid state ISE (De Marco et al., 2008; Ozdemir et al., 2013), despite the need of developing a controlled passive ion flow from the inner side of the sensing membrane towards the sample solution (Supporting Information, S1). To the best of our knowledge, this detection method has never been explored using a paper-based platform.

The foundation of the developed immunosensor is the transducing layer formed by the PVC-COOH polymer membrane and the PEDOT: PSS conductive ink. Fig. 3A shows that after membrane casting, the cellulose paper microfibers were completely and homogeneously coated by the polymer membrane. So, the chosen cocktail volume of $35 \mu \mathrm{L}$,

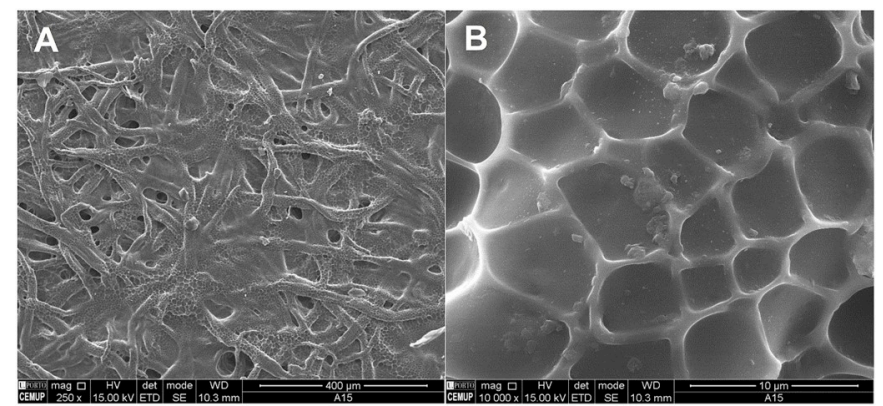

Fig. 3. SEM image of a working area of a PSE coated with the polymer membrane, using two different magnification levels: $250 \times(\mathrm{A})$ and $10000 \times(\mathrm{B})$.

such as the adopted enforcement procedure appears to be enough to waterproof the conductive paper (Ozdemir et al., 2013) and also to maintain the ion permeation capacity (Supporting Information, Fig. S1.2, S1.3). Besides, it was clear that polymer membrane was not only on the paper surface but also embedded into the pores and inter-cellulose fiber spaces, which will protect it from cracking (Fig. 3B) (Knoll et al., 1994). These features were observed in both sides of the conductive paper strip confirming the formation of a triple matrix ion sensitive membrane (Borchardt et al., 1995) and the possibility to use the developed PSE in combination with the surface blocking detection method. In further work, this PSE was used as the base sensor to assemble the two IMS represented in Fig. 2.

Regarding that the sensor membrane has to be suitable for bio-receptor immobilization, PVC-COOH was chosen because it provides free carboxylic groups to further modifications despite the known possible drawbacks in ion-sensor specificity (Xu and Bakker, 2009). Indeed PVC$\mathrm{COOH}$ has a hydrophilic surface by the carboxylic groups present, but its backbone is hydrophobic (Belegrinou et al., 2011; Pawlak et al., 2015). Therefore, these selectivity issues were thoughtfully minimized choosing a lipophilic marker ion with a privileged position in the Hofmeister series.

\subsection{Working sensing mechanism: proofing the theory}

The key point to establish and apply the proposed sensing mechanism is to reach a stable steady-state potential. In this specific condition, by mathematical deduction from Fick' s first law, any changes in EMF caused by an impediment in marker ion transfer kinetics can be correlated with a specific biorecognition event at aqueous layer (De Marco et al., 2008; Ozdemir et al., 2013). To explore this mechanism, it is necessary to promote a controlled passive flow of a marker ion through the sensing membrane. In conventional ISE operated in zero current potentiometry, the inner reference solution is the source of this ion flow towards the sample solution, but this electrode design poses several practical limitations (Cui et al., 2014; Ding et al., 2017; Lisak et al., 2015). Lisak, G et al. (Lisak et al., 2015) studied the possibility of using filter paper sampling coupled to potentiometric detection with all-solid-state and the sampling filter paper matrix may influence the response of the sensors towards some positive ions. Therefore, the response characteristics of the developed sensors to $\mathrm{TBA}^{+}$using this sampling strategy were evaluated (Supporting Information, Fig. S1.1) and the results showed that it is feasible to use a filter paper pad on the backside of the sensor to promote a $\mathrm{TBA}^{+}$flow. Fig. S1.3 in Supporting Information shows the changes in electrochemical signal triggered by the outwards flow surface disturbance caused by stir-effect on PSE, using two different TBA ${ }^{+}$concentrations. As expected, the accumulation of the marker ion next to sensor membrane amplifies the EMF step signal obtained, implying a subsequent improvement in immunosensor sensitivity. It was also verified, that the EMF step obtained due stir effect was correlated with the membrane conditioning time and the $\mathrm{TBA}^{+}$concentration in the PSE inner 
solution. Upon experimental optimization of these parameters, at least 1-day conditioning and a $14.4 \mathrm{mM}$ TBACl were selected for further work (Supporting Information, Fig. S4.1).

Additionally, diffusion profile studies suggests that the solution injected into the back of the PSE acts as the conventional electrode internal solution, in a manner assembled to a microfluidic or flow injection device, creating a passive diffusion profile established from the paper filter reservoir through the polymer membrane/PEDOT:PSS/ polymer membrane interfaces to the blank solution, since $20 \mathrm{~min}$ was enough to stabilization of EMF in a PB matrix (Supporting Information, Fig. S1.2). Besides the inherent increasing of the steps in the PSE construction, this procedure allowed us to use the zero-current potentiometry technique, that uses unparalleled instrumentation in terms of cost, complexity, and portability when compared with other techniques - like the pulstrode technique - capable to explore ion fluxes through an ESI membrane(Ding et al., 2013; Shvarev and Bakker, 2003).

\subsection{Immunosensing interface development}

In order to maximize the functionalization degree of the PSE transducing layer, some parameters of the two chosen IMS configurations were optimized: the EDC/NHS concentration used for coupling the $-\mathrm{COOH}$ groups of the polymer membrane to the capture antibody (IMS1); the PAMAM $\left(\mathrm{NH}_{2}\right)_{64}$ dendrimer concentration $(0.5 ; 2$ and $5 \%$, in IMS2); the glutaraldehyde crosslinker concentration and activation time (10 or $20 \mathrm{~min}$, IMS2) with such as the activation method used (two steps or one step, IMS2), the time used for antibody incubation $(2 \mathrm{~h}$ or "overnight", both IMS) and its concentration (1/50 or 1/100 of Ab $100 \mu \mathrm{gL}^{-1}$, IMS2); and BSA surface blocking step effect (both IMS) (Supporting Information, S2).

The results for IMS1 show that a slight increase of immunosensor sensibility was verified for longer overnight incubation comparing to $2 \mathrm{~h}$, although the benefit doesn't justify the increase in immunosensor preparation time. In IMS2, no significant advantage was verified using a higher antibody concentration, besides the increased surface area. The implementation of the BSA surface blocking step in both IMS studied increases the reproducibility between different modified electrodes. Although, decreased the amplitude of the signal obtained in correlation with the amount of ST added, confirming the surface blocking, greater uniformity of electrode modification despite the slight deceleration of the diffusion gradient through the modified membrane (Supporting Information Fig. S2.1). The optimized functionalization methods were fully described in section 2.2 above.

\subsection{Electrochemical impedance spectroscopy characterization}

Faradaic EIS characterization was conducted to monitor the impedance and resistance changes of the polymer surface along each modification step. All EIS data were modelled considering the Randle's equivalent circuit and fitted resorting to the electrochemical circle fit analysis tool from NOVA 1.7 in which charge transfer resistance (Rct) values were calculated and used as an indicator of modified electrode interface capability to transfer ions of the redox probe to the electrode surface.

The EIS studies performed allowed to confirm the success in the implementation of successive modification steps in both interfacial architectures (Supporting Information, Fig. S3.1A, B), herein an acceptable inter-electrode modification reproducibility was observed in all steps (RSV < 22.05\%). Additionally, for IMS1 was possible to confirm the successful antigen-antibody binging (Supporting Information Fig. S3.1A). This data was later corroborated by SEM images (Supporting Information, Fig. S3.1C).

Regarding IMS2 it was verified a higher change in Rct after the antibody and BSA immobilization steps comparing with results obtained for IMS1, suggesting an effective increase in the electrode area by the incorporation of the dendrimer in the IMS and a higher antibody

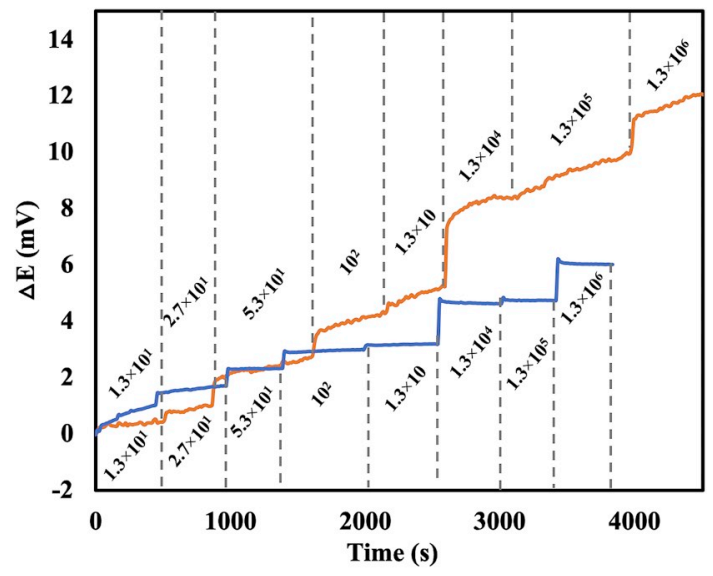

Fig. 4. Dynamic potentiometric measurements showing the response towards the indicated ST concentrations (cells $\mathrm{mL}^{-1}$ ) in $0.01 \mathrm{M}$ PB buffer. PBE modified with IMS1 (orange) and IMS2 (blue). (For interpretation of the references to color in this figure legend, the reader is referred to the Web version of this article.)

load. Despite those, a bad inter-electrode reproducibility of the antibody immobilization step (RSV $=52.64 \%$ ) was observed.

\subsection{Immunosensor performance}

The analytical performance of the proposed label-free immunosensor depends on the effectiveness of the biofunctionalization achieved and the integration efficiency of the proposed detection method in the modified PSE. Therefore, to look for the best functionalization method considering the detection method used, preliminary stir-effect experiments with PSE modified with IMS 1 and IMS2 were performed. Fig. S4.1 in Supporting Information shows that both IMS are sensitive to stir-effect after at least $4 \mathrm{~h}$ of internal conditioning, demonstrating that they are promising designs to develop a detection method based only on the surface blocking effect, but a lower potential difference was observed for IMS2, due to a denser barrier formed, creating a higher resistance to ionic flow comparing with IMS1 (see the previous section). Herein, successive additions of ST standard solutions $\left(10^{4}, 10^{6}, 10^{8}\right.$ cells $\left.\mathrm{mL}^{-1}\right)$ in a stirred PB matrix was carried out and typical dynamic potentiometric response curves of the immunosensors modified with IMS1 and IMS2 are presented in Fig. 4. After each addition, it was clear that the increase in $\mathrm{TBA}^{+}$concentration in the immunosensor surface vicinity caused by the passive flow blockage was electrochemically translated by an evident and rapid signal increase followed by a potential stabilization plateau.

Comparing the dynamic responses for the two IMS, lower noise level and sensitivity towards ST was observed for the IMS2 modified PSE. This observation can be attributed to changes in IMS structure, caused by the repulsion operated by non-connected dendrimer amine groups (that stays protonated at $\mathrm{pH}$ 7), that may transform the dendrimer in a flatter and dislike shape over the analysis time. As PAMAM $\left(\mathrm{NH}_{2}\right)_{64}$ dendrimer presents a relative flexibility "container properties", this setback is usually overcome simply by the use of a phosphate ionic background that gone into dendrimer pores creating an inert and stable conformation (Banyai et al., 2013; Scott et al., 2005; Taghavi Pourianazar et al., 2014). Although, should be noted that in this work was used a low concentration of ions in PB $\left(0.01 \mathrm{~mol} \mathrm{~L}^{-1}\right)$ in that may not be sufficient to block the positive charge (Lee et al., 2005).

Besides the inherent irreproducibility of IMS2 electrodes response anticipated by the EIS studies, a low sensitivity towards ST (slope of $1.0136 \mathrm{mV}$ per decade of cells concentration) was observed (Fig. 5 and Supporting Information, Fig. S4.3). Hereupon, by the need of a sensitive, feasible and accurate method, the IMS 1 was selected to integrate the optimized immunosensor design. Furthermore, experiments with a 


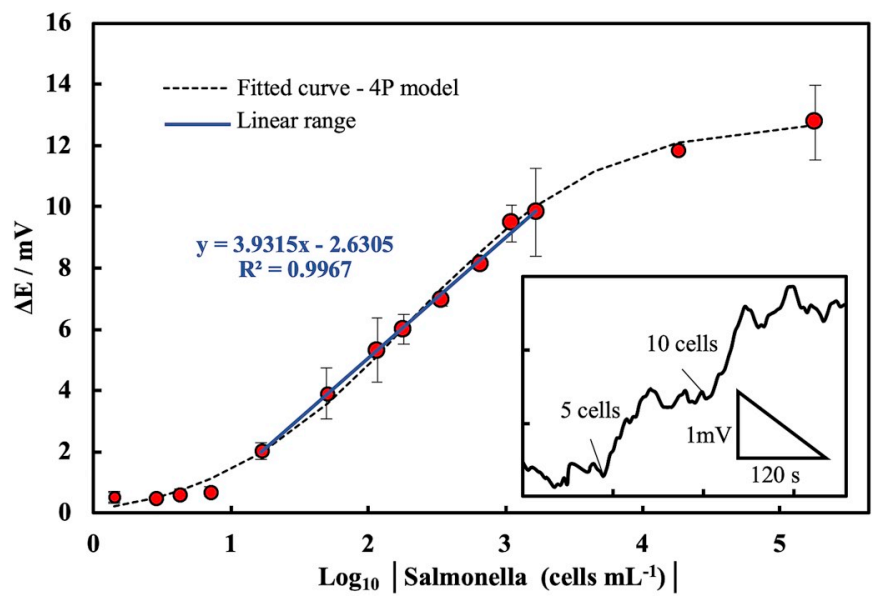

Fig. 5. Calibration curve representing the average values of $\triangle \mathrm{E}$ (measured potential - baseline potential $(3 \sigma= \pm 0.115 \mathrm{mV})$ ) with the standard deviation values (STD) of 3 different assays $(n=3)$; Dynamic potentiometric measure at low-level contamination of 5 and 10 cells (insert).

filtered matrix of Salmonella dilutions were made, to exclude possible non-specific interactions effects (Supporting Information, Fig.S4.2). None of the tested electrodes reacted to ST additions, confirming that the obtained EMF response is only derived by the biorecognition event between the antibody immobilized on the PSE surface and the ST cells added.

Fig. 5 represents the relation of EMF difference and the logarithm of ST concentration from 1 to $1.28 \times 10^{5}$ cells $\mathrm{mL}^{-1}$. The experimental data were fitted to a $4 \mathrm{P}$ sigmoidal model (dashed line in Fig. 5). In the concentration range from 12 to $12 \times 10^{3}$ cell mL $\mathrm{mL}^{-1}$ a linear response translated by equation EMF $(\mathrm{mV})=3.93 \times-2.63, \mathrm{R}^{2}=99.70 \%$ was achieved. Under these optimized experimental conditions, the calculated instrumental limit of detection was $115 \mu \mathrm{V}(3 \sigma=3 \times 38.20 \mu \mathrm{V})$, meaning that higher changes in EMF can be resolved. Nevertheless, even at low-level contamination experiments $(n=3)$, an unambiguous response was only obtained after inoculation of 5 cells $\mathrm{mL}^{-1}$ in a $5 \mathrm{~mL}$ sample volume, that corresponds to a $\Delta \mathrm{EMF}=296.0 \mu \mathrm{V}$ (inset of Fig. 5).

Considering the disposable character of the developed immunosensor it can be assumed that the obtained reproducibility (RSV $=12.10 \%, \mathrm{n}=3$ ) was satisfactory. However, it was tried to maximize the sustainability of the method, through the possibility of reusing of the electrode in several measures. Different chemical regeneration agents typically used for chemical regeneration $\mathrm{NaOH}$, Urea and Glycine-HCl) were employed after first time-use and the remaining immunosensor sensitivity after several regeneration cycles was evaluated (Supporting Information, S4.4) (Goode et al., 2015; Park et al., 2000). Urea solution successfully dissociates the antibody-antigen bond, although, after the second regeneration cycle, the response reduces to $46.00 \%$ and $50.49 \%$ of the initial response for $10^{4}$ and $10^{6} \mathrm{ST}$ cells, respectively.

Long-term stability of the method was tested in ready-to-use immunosensors that were stored at $4{ }^{\circ} \mathrm{C}$ in a moist atmosphere until use. After 3 weeks, the response falls to $64.20 \%$ of the initial (no longer times were tested).

Table S4.3 in Supporting Information compares the emerging methods in the literature - including other paper-based devices - for foodborne pathogens detection. Comparing with other electrochemical immunosensors (Altintas et al., 2018; Bhardwaj et al., 2017; Burrs et al., 2016; Khan et al., 2018) we can assume similar or higher time resolution of method, such as a higher range of quantification. Lower LOD values were attained with potentiometric aptamer resorting to the SWCNTs and graphene as ion-to-electron transducers (Hernández et al., 2014; Zelada-Guillén et al. 2009, 2010, 2013) and nonporous gold
Table 1

Recovery (\%) of Salmonella typhimurium (50, 100 and 1000 cells $\mathrm{mL}^{-1}$ ), in commercial apple juice samples.

\begin{tabular}{llll}
\hline Theoretical concentration & \multicolumn{2}{l}{ Recovery (\%) } \\
\hline (cells $\mathrm{mL}^{-1}$ ) & $\mathrm{n}$ & Mean & Coef.V (\%) \\
50 & 2 & 45.9 & $3.7 \%$ \\
100 & 2 & 63.0 & $29.6 \%$ \\
1000 & 2 & 53.0 & $11.0 \%$ \\
Mean & - & 54.0 & $15.0 \%$ \\
\hline
\end{tabular}

Coef.V $(\%)=$ coefficient of variation; $\mathrm{n}=$ number of samples.

modified platforms (Ranjbar et al., 2018), although this is the first time that such low LOD is reached for foodborne pathogens resorting to a potentiometric paper-based strip electrode. Some colorimetric methods have been used for the range reported, although the quantification at this level of infection can't be addressed (Hossain et al., 2012; Li et al., 2017; Srisa-Art et al., 2018; Suaifan et al., 2017a, b).

\subsection{Real sample analysis: proof of concept}

To guarantee a low strength ionic background, an efficient separation/purification technique was employed prophylactically prior to the analysis (see section 2.5 above). Next, the results of apple juice commercial real samples analysis were correlated with the calibration curve performed in PB. The recoveries \% obtained in samples inoculated with 50,100 and 1000 cells $\mathrm{mL}^{-1}$ of ST are shown in Table 1 . The recovery of ST in the range of concentrations 50 to 1000 cells $\mathrm{mL}^{-1}$ has an average value of $54.00 \%$, with a coefficient of variation of $15.00 \%$ like observed in the buffer $(12.00 \%)$. Besides the effectiveness of the pretreatment in sample purification, the modified protocol allows a poor recovery \% according to the previous values reported for (80-90\%) (Zelada-Guillén et al., 2010). Likely, the use of immunomagnetic separation coupled to the developed immunosensor may improve the recovery $\%$ in a simpler way, without increase total time of analysis.

\section{Conclusion and future prospects}

In this study, we present a label-free potentiometric immunosensor based on surface blocking principle and a zero current passive ion flux developed on a paper-based platform. The device consists of a paper strip ion selective electrode with a carboxylated PVC membrane integrated with a filter paper pad that acts as a reservoir for the internal solution. This design simultaneously provides a platform for antibody immobilization and is a simple and affordable methodology for controlling ionic flow through the polymer membrane.

As proof of concept, an immunosensor for Salmonella typhimurium was assembled using this platform and a limit of detection of 5 cells $\mathrm{mL}^{-1}$ was achieved in phosphate buffer. This prototype was applied in the quantification Salmonella in apple juice with an average recovery value of $54.00 \%$ and a coefficient of variation of $15.00 \%$, close to the value obtained in the phosphate buffer $(12.00 \%)$. Probably, the use of the immunomagnetic separation coupled to the developed immunosensor can improve the \% recovery more simply, without increasing the total time of analysis $(<1 \mathrm{~h})$.

The strategy presented in this work is a simple and accessible methodology to control ionic flow through the polymer membrane and shows potential for application to other bio-receptors. In addition to the optimization of the interface architecture with the immobilized bioreceptor, it is possible to design and optimize other devices inspired by the presented prototype, using different types of paper pad, selecting different ion selective polymer sensors and marker ions.

\section{CRediT authorship contribution statement}

Nádia F.D. Silva: Investigation, Writing - original draft. Cláudio 
M.R. Almeida: Investigation. Júlia M.C.S. Magalhães: Conceptualization, Supervision, Writing - review \& editing. Maria P. Gonçalves: Resources. Cristina Freire: Funding acquisition, Supervision. Cristina Delerue-Matos: Funding acquisition, Supervision, Writing - review \& editing.

\section{Acknowledgements}

This work received financial support from the European Union and National Funds (FCT, Fundação para a Ciência e Tecnologia) through projects UID/QUI/50006/2019 and Norte-01-0145-FEDER-000011RL1-QUALIFOOD. N.F.D Silva is grateful to FCT grant SFRH/BD/ $112414 / 2015$, financed by POPH-QREN-Tipologia 4.1-Formação Avançada, subsidized by FSE and MCTES.

\section{References}

Altintas, Z., Akgun, M., Kokturk, G., Uludag, Y., 2018. A fully automated microfluidicbased electrochemical sensor for real-time bacteria detection. Biosens. Bioelectron. $100,541-548$.

Apilux, A., Dungchai, W., Siangproh, W., Praphairaksit, N., Henry, C.S., Chailapakul, O., 2010. Lab-on-Paper with dual electrochemical/colorimetric detection for simultaneous determination of gold and iron. Anal. Chem. 82 (5), 1727-1732.

Armas, S.M., Manhan, A.J., Younce, O., Calvo-Marzal, P., Chumbimuni-Torres, K.Y., 2018. Ready-to-use single-strip paper based sensor for multiplex ion detection. Sensor. Actuator. B Chem. 255, 1781-1787.

Banyai, I., Keri, M., Nagy, Z., Berka, M., Balogh, L.P., 2013. Self-diffusion of water and poly(amidoamine) dendrimers in dilute aqueous solutions. Soft Matter 9 (5), 1645-1655.

Belegrinou, S., Menon, S., Dobrunz, D., Meier, W., 2011. Solid-supported polymeric membranes. Soft Matter 7 (6), 2202-2210.

Bhardwaj, J., Devarakonda, S., Kumar, S., Jang, J., 2017. Development of a paper-based electrochemical immunosensor using an antibody-single walled carbon nanotubes bio-conjugate modified electrode for label-free detection of foodborne pathogens. Sensor. Actuator. B Chem. 253, 115-123.

Booth, M.A., Kannappan, K., Hosseini, A., Partridge, A., 2015. In-depth electrochemica investigation of surface attachment chemistry via carbodiimide coupling. Langmuir 31 (29), 8033-8041.

Borchardt, M., Dumschat, C., Cammann, k., Knoll, M., 1995. Disposable ion-selective electrodes. Sensor. Actuator. B Chem. 25 (1), 721-723.

Burrs, S.L., Bhargava, M., Sidhu, R., Kiernan-Lewis, J., Gomes, C., Claussen, J.C., McLamore, E.S., 2016. A paper based graphene-nanocauliflower hybrid composite for point of care biosensing. Biosens. Bioelectron. 85, 479-487.

Canovas, R., Parrilla, M., Blondeau, P., Andrade, F.J., 2017. A novel wireless paper-based potentiometric platform for monitoring glucose in blood. Lab Chip 17 (14), 2500-2507.

Cao, L., Fang, C., Zeng, R., Zhao, X., Jiang, Y., Chen, Z., 2017. Paper-based microfluidic devices for electrochemical immunofiltration analysis of human chorionic gonadotropin. Biosens. Bioelectron. 92, 87-94.

Cui, J., Lisak, G., Strzalkowska, S., Bobacka, J., 2014. Potentiometric sensing utilizing paper-based microfluidic sampling. Analyst 139 (9), 2133-2136.

Currie, L.A., 1995. Nomenclature in evaluation of analytical methods including detection and quantification capabilities (IUPAC Recommendations 1995). Pure Appl. Chem. 1699.

De Marco, R., Ng, A., Panduwinata, D., 2008. Detecting biorecognition events at blocked interface polymeric membrane ion-selective electrodes using electrochemical impedance spectroscopy and atomic force microscopy. Electroanalysis 20 (3), 313-317.

Ding, J., Wang, X., Qin, W., 2013. Pulsed galvanostatic control of a polymeric membrane ion-selective electrode for potentiometric immunoassays. ACS Appl. Mater. Interfaces 5 (19), 9488-9493.

Ding, J., He, N., Lisak, G., Qin, W., Bobacka, J., 2017. Paper-based microfluidic sampling and separation of analytes for potentiometric ion sensing. Sensor. Actuator. B Chem. 243, 346-352.

European Commission (EC), E., 2005. Commission Regulation on Microbiological Criteria for Foodstuffs. Official Journal of the European Union, pp. 1-26.

European Food Safety Agency, European Centre for Disease Prevention, 2017. The European Union summary report on trends and sources of zoonoses, zoonotic agents and food-borne outbreaks in 2016. EFSA J. 15 (12), e05077.

European Food Safety Authority, European Centre for Disease Prevention, 2018. The European Union summary report on trends and sources of zoonoses, zoonotic agents and food-borne outbreaks in 2017. EFSA J. 16 (12), e05500.

Freitas, M., Viswanathan, S., Nouws, H.P.A., Oliveira, M.B.P.P., Delerue-Matos, C., 2014. Iron oxide/gold core/shell nanomagnetic probes and CdS biolabels for amplified electrochemical immunosensing of Salmonella typhimurium. Biosens. Bioelectron.
51, 195-200.

Fu, Y., Yuan, R., Tang, D., Chai, Y., Xu, L., 2005. Study on the immobilization of anti-IgG on Au-colloid modified gold electrode via potentiometric immunosensor, cyclic voltammetry, and electrochemical impedance techniques. Colloids Surfaces B Biointerfaces 40 (1), 61-66.

Goode, J.A., Rushworth, J.V.H., Millner, P.A., 2015. Biosensor regeneration: a review of common techniques and outcomes. Langmuir 31 (23), 6267-6276.

Gyurcsanyi, R.E., Vigassy, T., Pretsch, E., 2003. Biorecognition-modulated ion fluxes through functionalized gold nanotubules as a novel label-free biosensing approach. Chem. Commun. (20), 2560-2561.

Hernández, R., Vallés, C., Benito, A.M., Maser, W.K., Xavier Rius, F., Riu, J., 2014. Graphene-based potentiometric biosensor for the immediate detection of living bacteria. Biosens. Bioelectron. 54, 553-557.

Hossain, S.M.Z., Ozimok, C., Sicard, C., Aguirre, S.D., Ali, M.M., Li, Y., Brennan, J.D., 2012. Multiplexed paper test strip for quantitative bacterial detection. Anal. Bioanal. Chem. 403 (6), 1567-1576.

Hu, J., Stein, A., Bühlmann, P., 2016. A disposable planar paper-based potentiometric ion-sensing platform. Angew. Chem. Int. Ed. 55 (26), 7544-7547.

ISO, International Organization for Standardization, 1997. The Capability of Detection Part 1- Terms and Definitions. ISO, Genève, Switzerland.

ISO, International Organization for Standardization, 2003. The Capability of Detection Part 3: Methodology for Determination of the Critical Value for the Response Variable when No Calibration Data Are Used. ISO, Genève, Switzerland.

ISO, International Organization for Standardization, 2008. Capability O F Detection - Part 5: Methodology in the Linear and Non-linear Calibration Cases. ISO, Genève, Switzerland.

ISO, International Organization for Standardization, 2017. Microbiology of the Food Chain - Horizontal Method for the Detection, Enumeration and Serotyping of Salmonella - Part 1: Detection of Salmonella spp., ISO 6579-1 Geneva, Switzerland.

Khan, M.S., Misra, S.K., Dighe, K., Wang, Z., Schwartz-Duval, A.S., Sar, D., Pan, D., 2018. Electrically-receptive and thermally-responsive paper-based sensor chip for rapid detection of bacterial cells. Biosens. Bioelectron. 110, 132-140.

Knoll, M., Cammann, K., Dumschatt, C., Borchardt, M., Högg, G., 1994. Microfibre matrix-supported ion-selective PVC membranes. Sensor. Actuator. B Chem. 20 (1) 1-5.

Lee, C.C., MacKay, J.A., Frechet, J.M.J., Szoka, F.C., 2005. Designing dendrimers for biological applications. Nat. Biotechnol. 23 (12), 1517-1526.

Li, Z., Li, F., Xing, Y., Liu, Z., You, M., Li, Y., Wen, T., Qu, Z., Ling Li, X., Xu, F., 2017. Penon-paper strategy for point-of-care testing: rapid prototyping of fully written microfluidic biosensor. Biosens. Bioelectron. 98, 478-485.

Liébana, S., Lermo, A., Campoy, S., Cortés, M.P., Alegret, S., Pividori, M.I., 2009. Rapid detection of Salmonella in milk by electrochemical magneto-immunosensing. Biosens. Bioelectron. 25 (2), 510-513.

Lisak, G., Cui, J., Bobacka, J., 2015. Paper-based microfluidic sampling for potentiometric determination of ions. Sensor. Actuator. B Chem. 207, 933-939.

Liu, B., Du, D., Hua, X., Yu, X.Y., Lin, Y., 2014. Paper-based electrochemical biosensors: from test strips to paper-based microfluidics. Electroanalysis 26 (6), 1214-1223.

Narang, J., Singhal, C., Mathur, A., Khanuja, M., Varshney, A., Garg, K., Dahiya, T. Pundir, C.S., 2017. Lab on paper chip integrated with Si@GNRs for electroanalysis of diazepam. Anal. Chim. Acta 980, 50-57.

Novell, M., Parrilla, M., Crespo, G.A., Rius, F.X., Andrade, F.J., 2012. Paper-based ionselective potentiometric sensors. Anal. Chem. 84 (11), 4695-4702.

Ozdemir, M.S., Marczak, M., Bohets, H., Bonroy, K., Roymans, D., Stuyver, L., Vanhoutte, K., Pawlak, M., Bakker, E., 2013. A label-free potentiometric sensor principle for the detection of antibody-antigen interactions. Anal. Chem. 85 (9), 4770-4776.

Park, I.-S., Kim, W.-Y., Kim, N., 2000. Operational characteristics of an antibody-immobilized QCM system detecting Salmonella spp. Biosens. Bioelectron. 15 (3), 167-172.

Parrilla, M., Cánovas, R., Andrade, F.J., 2017. Paper-based enzymatic electrode with enhanced potentiometric response for monitoring glucose in biological fluids. Biosens. Bioelectron. 90, 110-116.

Pavithra, M., Muruganand, S., Parthiban, C., 2018. Development of novel paper based electrochemical immunosensor with self-made gold nanoparticle ink and quinone derivate for highly sensitive carcinoembryonic antigen. Sensor. Actuator. B Chem. 257, 496-503.

Pawlak, M., Mistlberger, G., Bakker, E., 2015. Concanavalin A electrochemical sensor based on the surface blocking principle at an ion-selective polymeric membrane. Microchimica Acta 182 (1), 129-137.

Ranjbar, S., Shahrokhian, S., Nurmohammadi, F., 2018. Nanoporous gold as a suitable substrate for preparation of a new sensitive electrochemical aptasensor for detection of Salmonella typhimurium. Sensor. Actuator. B Chem. 255, 1536-1544.

Rius-Ruiz, F.X., Crespo, G.A., Bejarano-Nosas, D., Blondeau, P., Riu, J., Rius, F.X., 2011. Potentiometric strip cell based on carbon nanotubes as transducer layer: toward lowcost decentralized measurements. Anal. Chem. 83 (22), 8810-8815.

Ruecha, N., Chailapakul, O., Suzuki, K., Citterio, D., 2017. Fully inkjet-printed paperbased potentiometric ion-sensing devices. Anal. Chem. 89 (19), 10608-10616.

Scott, R.W.J., Wilson, O.M., Crooks, R.M., 2005. Synthesis, characterization, and applications of dendrimer-encapsulated nanoparticles. J. Phys. Chem. B 109 (2), 692-704.

Shvarev, A., Bakker, E., 2003. Pulsed galvanostatic control of ionophore-based polymeric ion sensors. Anal. Chem. 75 (17), 4541-4550.

Silva, N.F.D., Magalhães, J.M.C.S., Freire, C., Delerue-Matos, C., 2018. Electrochemical biosensors for Salmonella: state of the art and challenges in food safety assessment. Biosens. Bioelectron. 99, 667-682.

Silva, N.F.D., Magalhães, J.M.C.S., Barroso, M.F., Oliva-Teles, T., Freire, C., DelerueMatos, C., 2019. In situ formation of gold nanoparticles in polymer inclusion membrane: application as platform in a label-free potentiometric immunosensor for Salmonella typhimurium detection. Talanta 194, 134-142. 
Srisa-Art, M., Boehle, K.E., Geiss, B.J., Henry, C.S., 2018. Highly sensitive detection of Salmonella typhimurium using a colorimetric paper-based analytical device coupled with immunomagnetic separation. Anal. Chem. 90 (1), 1035-1043.

Suaifan, G.A.R.Y., Alhogail, S., Zourob, M., 2017a. Paper-based magnetic nanoparticlepeptide probe for rapid and quantitative colorimetric detection of Escherichia coli O157:H7. Biosens. Bioelectron. 92, 702-708.

Suaifan, G.A.R.Y., Alhogail, S., Zourob, M., 2017b. Rapid and low-cost biosensor for the detection of Staphylococcus aureus. Biosens. Bioelectron. 90, 230-237.

Taghavi Pourianazar, N., Mutlu, P., Gunduz, U., 2014. Bioapplications of poly(amidoamine) (PAMAM) dendrimers in nanomedicine. J. Nanoparticle Res. 16 (4), 2342.

Teengam, P., Siangproh, W., Tuantranont, A., Henry, C.S., Vilaivan, T., Chailapakul, O., 2017. Electrochemical paper-based peptide nucleic acid biosensor for detecting human papillomavirus. Anal. Chim. Acta 952, 32-40.

Tian, T., Liu, H., Li, L., Yu, J., Ge, S., Song, X., Yan, M., 2017. Paper-based biosensor for noninvasive detection of epidermal growth factor receptor mutations in non-small cell lung cancer patients. Sensor. Actuator. B Chem. 251, 440-445.

Viswanathan, S., Rani, C., Ho, J.-a.A., 2012. Electrochemical immunosensor for multiplexed detection of food-borne pathogens using nanocrystal bioconjugates and MWCNT screen-printed electrode. Talanta 94, 315-319.

Wang, C.-C., Hennek, J.W., Ainla, A., Kumar, A.A., Lan, W.-J., Im, J., Smith, B.S., Zhao, M., Whitesides, G.M., 2016. A paper-based "Pop-up" electrochemical device for analysis of beta-hydroxybutyrate. Anal. Chem. 88 (12), 6326-6333.

$\mathrm{Xu}$, Y., Bakker, E., 2009. Ion channel mimetic chronopotentiometric polymeric membrane ion sensor for surface confined protein detection. Langmuir : ACS J. Surf. Colloid. 25 (1), 568-573.

Xu, Y., De Marco, R., Shvarev, A., Bakker, E., 2005. Reversible electrochemical monitoring of surface confined reactions at liquid-liquid interfaces by modulation of ion transfer fluxes. Chem. Commun. (24), 3074-3076.

Yang, F., Zuo, X., Fan, C., Zhang, X.-E., 2018. Biomacromolecular nanostructures-based interfacial engineering: from precise assembly to precision biosensing. Natl. Sci. Rev. 5 (2095-5138), 740.

Yoon, J.H., Kim, K.H., Bae, N.H., Sim, G.S., Oh, Y.-J., Lee, S.J., Lee, T.J., Lee, K.G., Choi, B.G., 2017. Fabrication of newspaper-based potentiometric platforms for flexible and disposable ion sensors. J. Colloid Interface Sci. 508, 167-173.

Zelada-Guillén, G.A., Riu, J., Düzgün, A., Rius, F.X., 2009. Immediate detection of living bacteria at ultralow concentrations using a carbon nanotube based potentiometric aptasensor. Angew. Chem. Int. Ed. 48 (40), 7334-7337.

Zelada-Guillén, G.A., Bhosale, S.V., Riu, J., Rius, F.X., 2010. Real-time potentiometric detection of bacteria in complex samples. Anal. Chem. 82 (22), 9254-9260.

Zelada-Guillén, G.A., Blondeau, P., Rius, F.X., Riu, J., 2013. Carbon nanotube-based aptasensors for the rapid and ultrasensitive detection of bacteria. Methods 63 (3), 233-238. 